\title{
COMMENTARY
}

\section{Is hydroxyethyl starch 130/0.4 safe?}

\author{
Nicolai Haase and Anders Perner* \\ See related research by Muller et al., http://ccforum.com/content/16/1/R34
}

\begin{abstract}
It is heavily debated whether or not treatment with hydroxyethyl starch 130/0.4 contributes to the development of acute kidney failure in patients with severe sepsis. In the previous issue of Critical Care, Muller and colleagues report no association between initial resuscitation with hydroxyethyl starch 130/0.4 and renal impairment in a cohort of septic patients. Can we then consider hydroxyethyl starch 130/0.4 a safe intervention? The answer is no - observational data should be interpreted with caution and should mainly be used to identify risks, while safety must be assessed in randomised clinical trials. With these factors in mind, Muller's data associate the use of vasopressors with poor outcome, underlining the need for further randomised clinical trials to assess the potential harmful effects of common interventions in the critically ill.
\end{abstract}

In the previous issue of Critical Care, Muller and colleagues present risk factors for renal dysfunction and renal replacement therapy in a cohort of 388 patients with severe sepsis or septic shock using multivariate analyses [1]. Of their results, the authors emphasise that there was no association between treatment with hydroxyethyl starch (HES) 130/0.4 in the first 24 hours of the study period and renal impairment.

Whether or not HES 130/0.4 causes acute kidney failure in septic patients is heavily debated. On one hand, the former starch solutions with higher molecular weight and substitution ratio caused acute kidney failure in septic patients when assessed in randomised clinical trials (RCTs) [2,3]. Also, the use of colloids has never been proven to decrease mortality in critically ill patients when compared with crystalloids [4]. On the other hand, the newer HES 130/0.4 is claimed to have a much better

*Correspondence: anders.perner@rh.regionh.dk

Department of Intensive Care, Copenhagen University Hospital - Rigshospitalet, Blegdamsvej 9, DK-2100 Copenhagen, Denmark safety profile. The volume-sparing effect of HES 130/0.4 seems much smaller than expected [5], however, and the safety and efficacy of this solution have not been adequately evaluated, as demonstrated in a recent systematic review [6].

The observational nature of the present study allows many explanations for the lack of association between HES treatment and poor renal outcome; for example, the authors probably underestimate the true incidence of renal dysfunction when using baseline creatinine from the current hospital admission, clinicians may have avoided HES treatment in patients at risk of developing renal failure, and the study power is too low. All of these factors tend to mask any harmful effect of HES treatment.

It is important to bear in mind that observational data should be used to identify potential adverse reactions of medical products, but should not be used to assess safety. Issues of safety must be assessed in well-powered RCTs with patient-important endpoints such as persistent organ failure, mortality and quality of life. HES 130/0.4 therefore cannot be considered safe even after the publication of the data by Muller and colleagues.

Better evidence will soon help clinicians decide on the use of HES 130/0.4 in sepsis. One larger trial has recently been published [5], and three large trials have finished the inclusion and their results will soon be reported [7-9]. Together, the results of these RCTs will elucidate the safety profile of HES 130/0.4 and inform us whether this starch should be used for septic patients. Until then, we believe that HES 130/0.4 should be given to septic patients only in the settings of RCTs as stated in the recently published European Society of Intensive Care Medicine guidelines for colloid therapy in intensive care [10].

Returning to the data presented by Muller and colleagues, another question emerges. The use of vasopressor was independently associated with a need for renal replacement therapy in their cohort of septic patients. Does this mean we should not use vasopressors for septic shock? Hopefully, in general, low validity of multivariate adjusting in ICU datasets with complex interventions contributes to this association. On the other hand, when choosing interventions in septic shock, the option often is either/or. If you give less fluid, you 
have to give more vasopressor. Fluid volume was not included in the analysis by Muller and colleagues, but again multivariate adjusting is unlikely to contribute to the answer to the question of the balance between fluid and vasopressor therapy. Here RCTs are urgently needed, in particular, as practice may be changing with more restrictive fluid therapy also in patients with sepsis [11].

Abbreviations

HES, hydroxyethyl starch; RCT, randomised clinical trial.

\section{Competing interests}

$\mathrm{AP}$ is the sponsor and $\mathrm{NH}$ is the coordinating investigator of the Scandinavian Starch for Severe Sepsis/Septic Shock Trial - a public-funded RCT investigating the effect of HES 130/0.4 versus crystalloid in patients with severe sepsis.

Published: 12 March 2012

\section{References}

1. Muller L, Jaber S, Molinari N, Favier L, Larché J, Motte G, Lazarovici S, Jacques L, Alonso S, Leone M, Constantin JM, Allaouchiche B, Suehs C, Lefrant JY; AzuRéa Group: Fluid management and risk factors for renal dysfunction in patients with severe sepsis and/or septic shock. Crit Care 2012, 16:R34.

2. Brunkhorst FM, Engel C, Bloos F, Meier-Hellmann A, Ragaller M, Weiler N, Moerer O, Gruendling M, Oppert M, Grond S, Olthoff D, Jaschinski U, John S, Rossaint R, Welte T, Schaefer M, Kern P, Kuhnt E, Kiehntopf M, Hartog C, Natanson C, Loeffler M, Reinhart K; German Competence Network Sepsis (SepNet): Intensive insulin therapy and pentastarch resuscitation in severe sepsis. NEngl J Med 2008, 358:125-139.

3. Schortgen F, Lacherade JC, Bruneel F, Cattaneo I, Hemery F, Lemaire F, Brochard L: Effects of hydroxyethylstarch and gelatin on renal function in severe sepsis: a multicentre randomised study. Lancet 2001, 357:911-916.

4. Perel P, Roberts I: Colloids versus crystalloids for fluid resuscitation in critically ill patients. Cochrane Database Syst Rev 2011, 3:CD000567.
5. Effects of Voluven on Hemodynamics and Tolerability of Enteral Nutrition in Patients with Severe Sepsis [http://clinicaltrials.gov/ct2/show/ NCT00464204]

6. Hartog CS, Kohl M, Reinhart K: A systematic review of third-generation hydroxyethyl starch (HES 130/0.4) in resuscitation: safety not adequately addressed. Anesth Analg 2011, 112:635-645.

7. Crystalloid versus Hydroxyethyl Starch Trial (CHEST) Management Committee: The Crystalloid versus Hydroxyethyl Starch Trial: protocol for a multi-centre randomised controlled trial of fluid resuscitation with $6 \%$ hydroxyethyl starch (130/0.4) compared to $0.9 \%$ sodium chloride (saline) in intensive care patients on mortality. Intensive Care Med 2011, 37:816-823.

8. Perner A, Haase N, Wetterslev J, Aneman A, Tenhunen J, Guttormsen AB, Klemenzson G, Pott F, Bødker KD, Bådstøløkken PM, Bendtsen A, Søe-Jensen P, Tousi H, Bestle M, Pawlowicz M, Winding R, Bülow HH, Kancir C, Steensen M, Nielsen J, Fogh B, Madsen KR, Larsen NH, Carlsson M, Wiis J, Petersen JA, Iversen S, Schøidt O, Leivdal S, Berezowicz P, et al:: Comparing the effect of hydroxyethyl starch 130/0.4 with balanced crystalloid solution on mortality and kidney failure in patients with severe sepsis (6S Scandinavian Starch for Severe Sepsis/Septic Shock trial): study protocol, design and rationale for a double-blinded, randomised clinical trial. Trials 2011, 12:24

9. BaSES Trial: Basel Starch Evaluation in Sepsis [http://clinicaltrials.gov/ct2/ show/NCT00273728]

10. Reinhart $K$, Perner A, Sprung $C L$, Jaeschke $R$, Schortgen $F$, Johan Groeneveld $A B$, Beale R, Hartog CS: Consensus statement of the ESICM task force on colloid volume therapy in critically ill patients. Intensive Care Med 2012. [Epub ahead of print]

11. Hilton AK, Bellomo R: A critique of fluid bolus resuscitation in severe sepsis. Crit Care 2012, 16:302.

doi:10.1186/cc11200

Cite this article as: Haase N, Perner A: Is hydroxyethyl starch 130/0.4 safe? Critical Care 2012, 16:116. 